\title{
Dynamic Coded Cooperation using Multiple Turbo Codes in Wireless Relay Networks
}

\author{
Koji Ishibashi*, Koji Ishii, and Hideki Ochiai \\ ${ }^{*}$ Corresponding Author: Koji Ishibashi \\ Department of Electrical and Electronic Engineering \\ Faculty of Engineering, Shizuoka University \\ email: koji@ieee.org \\ EDICS: WIN-CONT
}

Manuscript submitted on Nov. 6, 2009; revised Mar. 30, 2010 and June 19, 2010.

This paper was presented in part at the IEEE Vehicular Technology Conference (VTC2009)

Fall, Anchorage, Alaska, USA, Sept. 2009 and at the IEEE International Conference on

Communications (ICC2010), Cape Town, South Africa, May 2010.

This work is supported by Strategic Information and Communications R\&D Promotion

Programme (SCOPE), Ministry of Internal Affairs and Communications, Japan.

\begin{abstract}
In this paper, we propose a dynamic coded cooperation using multiple turbo codes in wireless relay networks where both relay and destination are equipped with a sensing device and the relay is assumed to determine autonomously if it should cooperate or not. We first elucidate the fact that the puncturing patterns of the multiple turbo codes determine the achievable diversity order by deriving their pairwise error probabilities (PEP). This is followed by a derivation of a simple power detector at the destination that can estimate a precise duration of cooperation phase upon decoding information. Furthermore, we investigate the impact of process delay due to cognitive processing at the relay, which results in the loss of diversity gain, and propose a simple null padding as its countermeasure. Computer simulations show that our proposed approach is able to achieve a full diversity and the frame error rate (FER) performance superior to that of conventional cooperative communications that make use of turbo coding structure even in practical scenarios.
\end{abstract}

\section{Index Terms}

Transmit diversity, cooperative communications, dynamic decode-and-forward (DDF), multiple turbo codes, power detection, distributed turbo codes

\section{INTRODUCTION}

Recently, cognitive radio has gained much attention as a new paradigm of wireless communications, which promises an enhanced utilization of the limited spectrum resource. Cognitive radio technology enables one to autonomously recognize underutilized frequency bands called white spaces. The terminals with this advanced technology (secondary users) thus can dynamically communicate each other without interfering with licensed radio services (primary users) [1-3]. 
In order to exploit the limited transmission opportunity, it is essential for the cognitive radio network to increase its bandwidth efficiency [4].

Unfortunately, in typical wireless channels, a multipath fading significantly reduces instantaneous communication capacity for a given average transmission power and hinders the efficient use of available limited white spaces. Results on pioneering works of space time coding (STC) have shown that the use of antenna arrays at the transmitter and receiver can significantly mitigate channel impairments even in flat fading channels $[5,6]$. However, for many applications with mobile devices, deployment of multiple antennas at each terminal is too costly to implement due to severe constraints on both size and power consumption of analog devices. To overcome this issue, cooperative (or collaborative) diversity approaches have been proposed [7-11], which demonstrate a potential to achieve a diversity gain or enhance the capacity of wireless systems without deploying multiple antennas at the transmitter. In most of early work on decode and forward (DF) protocols, the source and relay use an identical channel code. Also, it has been shown that the performance of DF protocol can be improved by using a different channel code at the relay, which is referred to as coded cooperation in the literature. In [9,11], the application of turbo codes to coded cooperation has been investigated and it was demonstrated that the performance can be improved by judiciously deploying channel code at the relay. These approaches, however, require two communication channels or larger bandwidth (i.e., source to destination and relay to destination) to meet a half-duplex constraint of communicating terminals. This constraint reduces an overall bandwidth efficiency of wireless networks.

To mitigate this inherent loss of bandwidth efficiency, dynamic decode-and-forward (DDF) has been recently proposed in the literature [12-14]. In this protocol, if the receivers (i.e., relay and destination) have a knowledge of network configuration, the relay can dynamically superimpose cooperating signals on the original signal from the source using the same channel. As a result, the destination can achieve the diversity gain without extra channel resources. Although the existence of such bandwidth efficient cooperative code has been proved from an information theoretical viewpoint [12-14], a practical approach for constructing such codes has not been proposed. In [15], an implementation of DDF based on rateless codes, also known as Fountain codes [16], has been proposed. Further results on this issue can be found in the recent work [17]. 


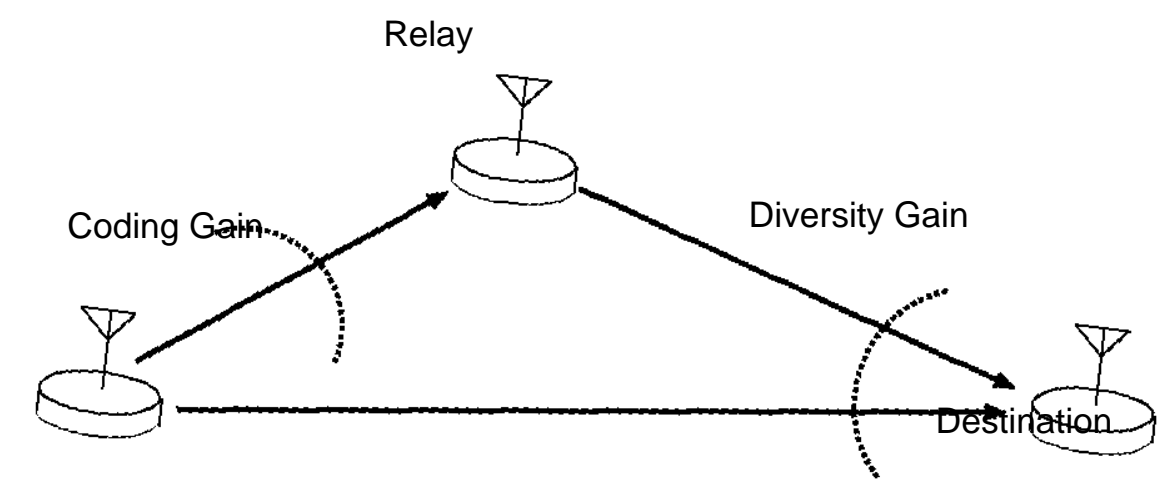

Source

Fig. 1. Cooperative (collaborative) communications model.

Finally, another implementation of DDF based on lattice and permutation codes, derived from universally decodable matrices, has been studied in [18]. These conventional works, however, have considered neither the practical feasibility of DDF techniques nor the effect of process delay at the relay terminal due to decoding.

In this paper, we propose a simple and feasible DDF cooperative protocol using multiple turbo codes in wireless relay networks, which is referred to as a dynamic coded cooperation throughout the paper. It is worth noting that dynamic coded cooperation inherently relies on a framework of cognitive radio technology since it can be implemented only if the relay is equipped with a sensing device recognizing the source's transmission and the destination can autonomously recognize whether the relay is cooperating or not [19]. The main contributions of the paper are summarized as follows:

- The design criterion of rate compatible turbo codes that is suitable for the DDF protocol proposed in [12] is investigated based on pairwise error probability (PEP) analysis.

- The design of a simple power detector for the dynamic coded cooperation is studied and its performance is theoretically analyzed.

- We extend the model of [12] to investigate the effect of process delay at the relay and show that this process delay degrades the diversity gain at the destination. Also, the simple null padding is proposed as its countermeasure.

Following the framework of [12], we focus on the DDF protocol for the half-duplex, single- 
relay, and single-antenna case as shown in Fig. 1. In this case, the diversity gain cannot be achieved at the relay since the frame is disturbed by only one channel coefficient if flat fading channels are assumed. Thus, the coding gain dominates the decoding error performance at the relay. On the other hand, at the destination, the diversity gain dominates the overall performance rather than the coding gain. Based on this observation, we derive a design criterion of rate compatible turbo codes by analyzing the PEP and propose a design guideline of turbo codes to achieve both full diversity and increasing coding gain.

Upon decoding at the destination in the DDF cooperation, the receiver has to estimate the precise timing of when the cooperation phase has been started since the destination does not generally know a prior knowledge of relay's decision. In [18], generalized likelihood ratio test (GLRT) rule has been proposed to estimate this timing, which jointly decodes the relay decision time and the information. In this paper, we focus on a design of a power detector for the dynamic coded cooperation since the power detector is often used in mobile devices to simply detect existences of other devices or systems especially in cognitive radio networks [3].

Finally, we investigate the impact of process delay due to cognitive processing at the relay, which has not been considered in the literature even though this effect cannot be avoided in practice. Existence of processing delay hinders the relay from immediately transmitting its cooperating signals even if it decodes the information correctly. Thus, this process delay may affect the overall performance of the dynamic coded cooperation. To overcome this issue, a simple null padding is proposed.

This paper is organized as follows. In Section II, we briefly describe the system model assumed in this paper. The design of turbo codes for the dynamic coded cooperation is described in Section III. In Section IV, the performance of a simple power detector at the destination is investigated to estimate the duration of listening phase for decoding the information at the destination. In Section $\mathrm{V}$, computer simulations are performed to exemplify the analytical results and investigate the performance of dynamic coded cooperation in practical scenarios. Finally, Section VI concludes this work. 


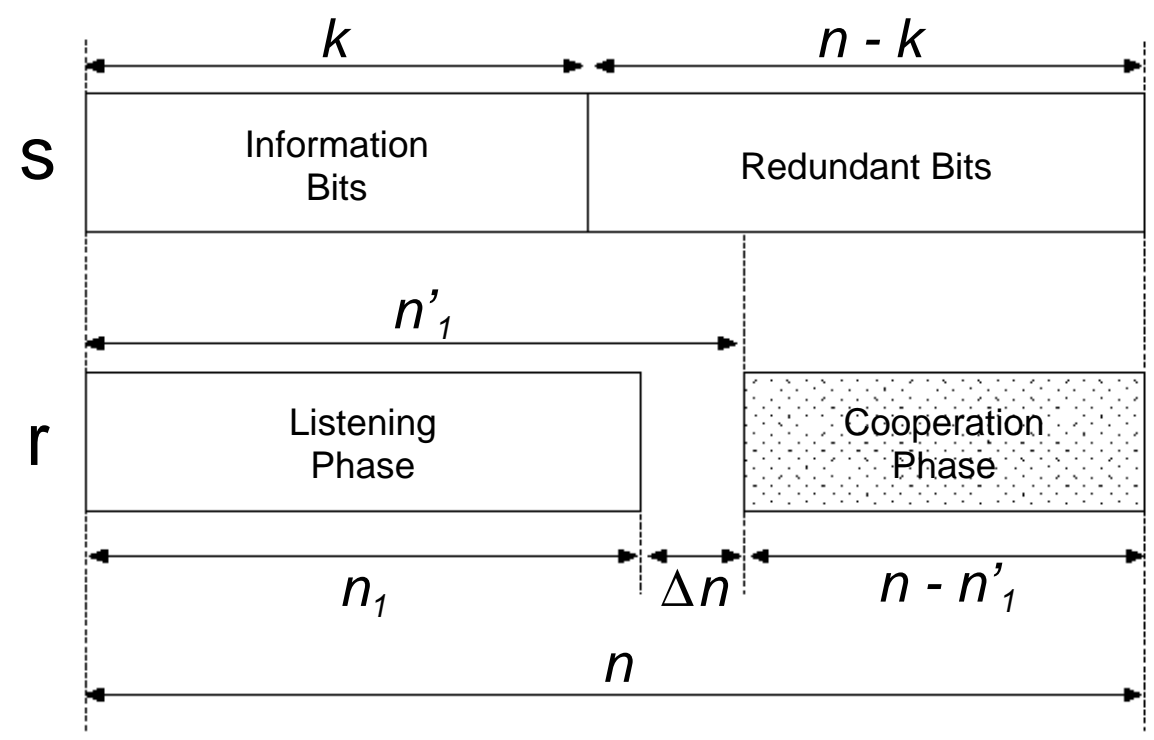

Fig. 2. The frame model of the dynamic coded cooperation.

\section{System Model}

In this paper, we consider a network consisting of three terminals denoted as source (s), relay (r), and destination (d). Each terminal is equipped with a single antenna and the relay is subject to the half-duplex constraint. Thus, we assume that while listening to the channel, the relay may not transmit. We also assume that the source transmits a codeword of symbol length $n$. In [12], it is proposed that the relay listens during first $n_{1}$ symbol length of the codeword and then transmits a part of the codeword of symbol length $n-n_{1}$. In this paper, we consider the process delay at the relay mainly due to the decoding, which cannot be avoided in practice. We therefore assume that the relay is unable to immediately transmit cooperating signals upon the partial reception of source transmission even if the relay decodes the information correctly. Hence, we consider the model that the relay requires $\Delta n$ symbol duration to perform the decoding and cannot transmit signals during this duration as illustrated in Fig. 2. Consequently, the relay listens during $n_{1}$ symbol length (listening phase) and transmits a part of the codeword of symbol length $n-n_{1}-\Delta n=n-n_{1}^{\prime}$ (cooperation phase).

Let the transmitted signals from the source terminal be denoted by $\mathbf{x}_{s}=$ $\left[x_{s}(1), x_{s}(2), \cdots, x_{s}(n)\right]$. Each element of $\mathbf{x}_{s}$ is assumed to be chosen from a set of $M$-level 
phase shift keying (PSK) symbols. Thus,

$$
x_{s}(i)=e^{j \phi_{i}}
$$

where $i=1, \cdots, n$ and $\phi_{i} \in\{2 \pi l / M \mid l=0,1, \cdots, M-1\}$. Without loss of generality, we assume that all transmit antennas have unit average power during their respective transmission phases. The received signals at the destination and the relay terminals are denoted by $\mathbf{y}_{s d}=$ $\left[y_{s d}(1), y_{s d}(2), \cdots, y_{s d}\left(n_{1}^{\prime}\right)\right]$ and $\mathbf{y}_{s r}=\left[y_{s r}(1), y_{s r}(2), \cdots, y_{s r}\left(n_{1}\right)\right]$, respectively. Then during the listening phase, the $i$ th received signals are given by

$$
\begin{aligned}
& y_{s d}(i)=h_{s d} \sqrt{g_{s d}} x_{s}(i)+z_{d}(i) \\
& y_{s r}(i)=h_{s r} \sqrt{g_{s r}} x_{s}(i)+z_{r}(i),
\end{aligned}
$$

where $z_{d}(i)$ and $z_{r}(i)$ are independent complex additive white Gaussian noise (AWGN) with variance $\sigma^{2}, g_{s d}$ and $g_{s r}$ are the corresponding path-loss coefficients, and $h_{s d}$ and $h_{s r}$ are the corresponding complex fading coefficients, assumed to be constant during transmission of each codeword. All the fading coefficients are assumed to be uncorrelated and circularly-symmetric complex Gaussian random variables with zero mean and unit variance. During the cooperation phase, i.e., $n_{1}^{\prime}+1 \leq i \leq n$, the relay transmits $M$-PSK signals denoted by $\mathbf{x}_{r}=\left[x_{r}\left(n_{1}^{\prime}+\right.\right.$ $\left.1), x_{r}\left(n_{1}^{\prime}+2\right), \cdots, x_{r}(n)\right]$, if and only if the relay successfully decodes the information in listening phase. Then, we have

$$
y_{c}(i)=h_{s d} \sqrt{g_{s d}} x_{s}(i)+h_{r d} \sqrt{g_{r d}} x_{r}(i)+z_{d}(i)
$$

where $\mathbf{y}_{c}=\left[y_{c}\left(n_{1}^{\prime}+1\right), y_{c}\left(n_{1}^{\prime}+2\right), \cdots, y_{c}(n)\right]$ is the received signals at the relay, $g_{r d}$ is the path-loss coefficient between the relay and destination, and $h_{r d}$ is the corresponding complex fading coefficient. We further assume that the channel state information (CSI) is available only at the receiver side; both $h_{s d}$ and $h_{r d}$ are known at the destination but only $h_{s r}$ is known at the relay.

For simplicity, we assume that the average receive signal to noise ratio (SNR) between the relay and destination $\Gamma_{r d}$ is identical with that between the source and destination $\Gamma_{s d}$ since we focus on the scenario where the relay is closely located to the source. Furthermore, in practice, 
the nearby terminals are easier to cooperate than those located apart. Following [12], we model this proximity by introducing relative path-loss gain $G$ defined as

$$
G \triangleq 10 \log _{10}\left[\frac{g_{s r}}{g_{s d}}\right] .
$$

Thus, the SNR between the source and relay can be given as $\Gamma_{s r}=\Gamma_{s d}+G$ in $\mathrm{dB}$. Without loss of generality, we restrict our interests to the case where $G \geq 0$ throughout the paper.

\section{A. Protocol Description}

1) Conventional Approaches: We here briefly explain two conventional cooperative approaches. In the case of DF cooperation with repetition coding, the source transmits the information to both the relay and destination (i.e., broadcasting) in the listening phase. The relay attempts to decode the information and, if it can correctly decode the information, the relay transmits the identical data frame to the destination in the cooperation phase. At the destination, two received frames are combined before decoding by using maximum ratio combining (MRC) to obtain diversity gain efficiently. Thus, in this approach, the bandwidth efficiency becomes half compared with the direct transmission without relay.

In the case of conventional coded cooperation $[9,11]$, the source transmits the coded information to both the relay and destination similar to DF cooperation. Then, the relay attempts to decode the information. In the cooperation phase, the relay transmits the additional redundant bits to the destination where its length is half compared with the original data frame [9]. Although the conventional coded cooperation can improve the bandwidth efficiency compared with DF cooperation with repetition coding, the bandwidth efficiency becomes lower than that of the simple direct transmission without relay.

2) Dynamic Coded Cooperation: In comparison with the conventional approaches, the dynamic coded cooperation, originally proposed in [12], does not decrease bandwidth efficiency. This cooperative protocol allows that the relay adjusts the cooperation phase based on the quality of the channel between the source and relay. In order to achieve this dynamic adaptation with a feasible channel code, we propose the use of rate compatible turbo codes [20]. Rate compatible turbo codes can be simply constructed by eliminating specific redundant bits of the codeword of original binary turbo codes (which is referred to as a mother code in this paper) with rate $R$. 
Since the original turbo code is a systematic code, the codeword can be divided into information bits and redundancy bits. Prior to the cooperative transmission, the source should choose its own coding rate $R_{c}>R$ by puncturing parity bits and punctured parity bits are assigned to the relay similar to the conventional coded cooperation scheme [11]. Then, coded bits transmitted from the source are arranged in a decreasing order of coding rate similar to incremental redundancy. Therefore, information bits are placed in the head of the frame.

Based on this frame model, we describe our feasible diversity enhancement protocol. If the channel $h_{s r}$ is sufficiently good, the relay adjusts the length of listening phase $n_{1}$ to the length of information bits $k$. After receiving $k$ information bits, the relay reencodes the information sequence and transmits to the destination during $n-k-\Delta n$ transmissions. On the other hand, if the channel $h_{s r}$ is moderately good, the relay adjusts $n_{1}$ to the codeword length as shown in Fig. 2. The coding rate between the source and relay $R_{s}$ and the Resultant coding rate can be defined by the actual value of $n_{1}$. After receiving the punctured codeword, the relay decodes the information. Finally, the relay would transmit the parity bits punctured from the original codeword in $n-n_{1}^{\prime}$ transmissions.

Compared with the conventional fixed-rate coded cooperation, this adaptive rate control can decrease the probability that the relay does not successfully decode the information and thus might improve the overall performance. However, in practice, it is infeasible that the relay attempts to decode the information every time it receives one symbol. Thus, the fractional reception of the codeword is considered. We assume that the redundant bits allocated to the source are divided into $K$ fractions. Then $n_{1}$ can be chosen by

$$
n_{1}=k+n_{b} \times l,
$$

where $l=0,1, \cdots, K-1$ and

$$
n_{b}=\frac{n-k}{K} .
$$

If the integer $l$ equals $K$, the relay does not cooperate, resulting in a conventional single transmission. This fractional reception mitigates the difficulty of estimating $n_{1}^{\prime}$ at the receiver even by using the simple power detection as will be discussed in Section IV. 
Also, we assume that the relay continues to receive and store the symbols of the next fraction even if the relay is in the state of decoding process. This parallel signal processing can be performed by using a buffer memory holding outputs of the matched filter at every Nyquist interval.

Finally, we further assume that the effect of clock and carrier synchronization errors are negligible for simplicity. Thus, the three terminals are perfectly synchronized at the symbol level.

\section{Code Design of Dynamic Coded CoOperation}

In this section, we derive the PEP of rate compatible turbo code based on the aforementioned model and investigate the coding design that can achieve the full diversity gain in dynamic coded cooperation. We elucidate the necessity of using multiple turbo codes for enhancing the probability of cooperation.

\section{A. Pairwise Error Probability}

In the cooperation phase, the received signal $y_{c}(i)$ is given by the superposition of signals from source and relay as shown in (4). Exact theoretical performance analysis of the dynamic coded cooperation for a given specific code is challenging and beyond the scope of this paper. Nevertheless, the performance becomes analytically tractable if we ignore the mutual correlation between the source and relay. Therefore, we assume that the source and relay use orthogonal channels only for the purpose of subsequent analysis. This optimistic assumption guarantees that the interference between $\mathbf{x}_{s}$ and $\mathbf{x}_{r}$ does not occur in cooperation phase. Consequently, the resulting performance serves as a lower bound of the performance achieved by our proposed dynamic coded cooperation. Furthermore, for simplicity, we consider the case where the relay always decodes the information correctly by receiving only $k$ information bits. Although the lower bound with these ideal assumptions turns out to be considerably loose, it still provides a useful analytical guideline on how to obtain full diversity gain by allocating a portion of a codeword to the relay in both dynamic and conventional turbo coded cooperation, which has not been investigated in the literature $[9,11]$. 


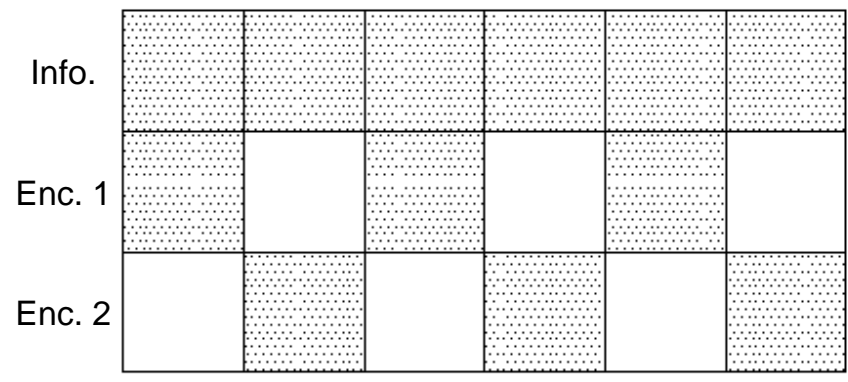

(a) Conventional Puncturing

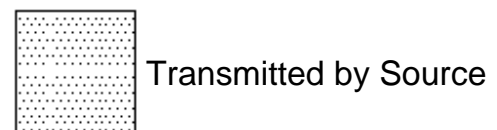

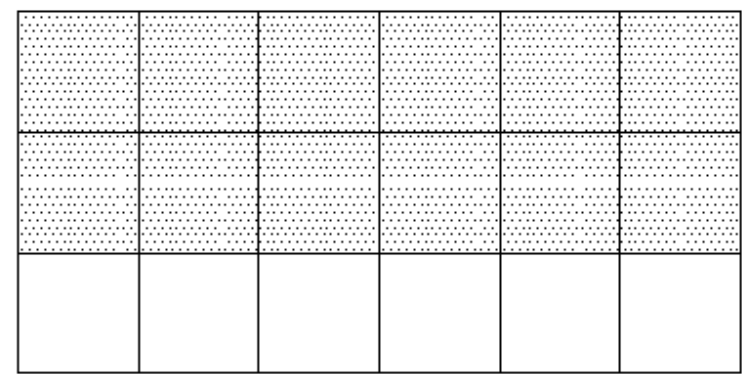

(b) Componentwise Puncturing

Transmitted by Relay

Fig. 3. Puncturing patterns for rate compatible turbo code.

Assuming orthogonal source and relay channels, the PEP for a coded system is the probability of detecting an erroneous codeword e when codeword $\mathbf{c}$ is transmitted and can be bounded, for high SNR, by [11]

$$
\operatorname{Pr}(\mathbf{c} \rightarrow \mathbf{e}) \leq \frac{1}{2}\left(\frac{1}{1+w_{1} \Gamma_{s d}}\right)\left(\frac{1}{1+w_{2} \Gamma_{r d}}\right),
$$

where $w_{1}$ and $w_{2}$ are the Hamming weights of the codewords transmitted by source and relay, respectively.

\section{B. Design of Rate Compatible Turbo Code in Full Diversity Criterion}

Based on the previous observation, the design of rate compatible turbo code for the proposed approach is described here. From (8), the full diversity order of two is achieved if and only if both $w_{1}$ and $w_{2}$ are non-zero. This full-diversity condition is critical for the design of the additional parity bits allocated to the relay (i.e., puncturing matrix).

Without loss of generality, we here consider the original turbo code, which consists of two recursive systematic convolutional (RSC) encoders. Specifically, the coding rate of the mother code is assumed to be $R=1 / 3$ and the coding rate after puncturing, $R_{c}$, is assumed to be half in the following. Then, for a given error event, let $d_{I}, d_{1}$, and $d_{2}$ indicate the Hamming weights of information bits, parity bits from the first RSC encoder, and parity bits from the second RSC encoder, respectively. In the dynamic coded cooperation, information bits are always transmitted 
by the source. Thus, for using any puncturing pattern, $w_{1}$ in (8) satisfies the following inequality:

$$
w_{1} \geq d_{I} \geq 2
$$

since the RSC component encoders have the minimum error event with the minimum information weight 2 [20]. Consequently, in order to achieve the full diversity, the following condition should be satisfied.

Full Diversity Condition: Consider the pairwise error event that an erroneous codeword e is chosen at the decoder instead of a correct codeword c, and let $w_{2}$ indicate the Hamming weight transmitted by relay as in (8). Then, if $w_{2}$ becomes non-zero, the dynamic coded cooperation can achieve the full diversity.

By inspection of this full diversity condition, the event of the codeword with $w_{2}=0$ corresponds to the loss in terms of diversity order. Thus, in the following, the occurrence of the event $w_{2}=0$ is analyzed for given puncturing patterns. Two types of commonly used puncturing patterns are considered in this paper, i.e., conventional puncturing and componentwise puncturing as depicted in Fig. 3. With the conventional puncturing, the source would transmit the information bits and the redundant bits of both first and second encoders by turns. On the other hand, with the componentwise puncturing the source would transmit the information bits and redundant bits generated by the first component encoder. The relay would transmit the residual bits in both cases.

1) Conventional Puncturing: Let $N$ denote the path length of the arbitrary error event and $m_{1}$ denote the minimum weight generated by the first RSC encoder. Then, assuming the uniform interleaver [21], we may obtain the asymptotic bound of the probability that $w_{2}$ is zero as

$$
\operatorname{Pr}\left(w_{2}=0\right) \leq \frac{1}{N\left(N-m_{1}\right)} \sum_{d_{1}=m_{1}}^{N} \sum_{d_{2}=0}^{N} \frac{\left(\begin{array}{l}
\frac{N}{2} \\
d_{1}
\end{array}\right)\left(\begin{array}{l}
\frac{N}{2} \\
d_{2}
\end{array}\right)}{\left(\begin{array}{l}
N \\
d_{1}
\end{array}\right)\left(\begin{array}{l}
N \\
d_{2}
\end{array}\right)},
$$

for even $N$ and

$$
\operatorname{Pr}\left(w_{2}=0\right) \leq \frac{1}{N\left(N-m_{1}\right)} \sum_{d_{1}=m_{1}}^{N} \sum_{d_{2}=0}^{N} \frac{\left(\begin{array}{c}
\frac{N+1}{2} \\
d_{1}
\end{array}\right)\left(\begin{array}{c}
\frac{N-1}{2} \\
d_{2}
\end{array}\right)+\left(\begin{array}{c}
\frac{N-1}{2} \\
d_{1}
\end{array}\right)\left(\begin{array}{c}
\frac{N+1}{2} \\
d_{2}
\end{array}\right)}{2\left(\begin{array}{c}
N \\
d_{1}
\end{array}\right)\left(\begin{array}{c}
N \\
d_{2}
\end{array}\right)},
$$

for odd $N$. Obviously, the upper bound of $\operatorname{Pr}\left(w_{2}=0\right)$ cannot be made zero for moderate length of $N$. Therefore, conventional puncturing cannot guarantee the full diversity gain even if all assigned bits are transmitted by the relay. 
2) Componentwise Puncturing: When the componentwise puncturing is used, we have $w_{1}=$ $d_{I}+d_{1}$ and $w_{2}=d_{2}$. Therefore, in this case, the full diversity of order two can be achieved.

However, considering the process delay defined in Section II-A, the relay cannot transmit the entire codeword generated by the second component encoder and thus the full diversity condition is not necessarily guaranteed. Therefore, in this case, the dynamic coded cooperation may degrade achievable diversity gain even with a small process delay. A simple countermeasure to avoid this impairment is padding $\Delta n$ null symbols into the position after information bits in the source's frame. Although this approach leads to a slight loss of bandwidth efficiency, it can be made negligibly small compared to the increase of bandwidth efficiency achieved by the dynamic coded cooperation. These issues will be analyzed quantitatively through numerical results in Section V.

\section{Design of Rate Compatible Turbo Code in Coding Gain Criterion}

The previous subsection shows that componentwise puncturing should be used to obtain the full diversity at the destination. At the relay, the frame is disturbed by only one channel coefficient and thus the coding gain dominates the decoding error performance. However, if componentwise puncturing and rate $1 / 3$ mother code with two component RSC codes are assumed, iterative decoding cannot be performed at the relay since the source does not transmit the redundant bits generated by the second RSC. Therefore, the coding gain becomes relatively small and the efficient performance improvement cannot be achieved in the dynamic coded cooperation.

Thus, in order to overcome this design issue, we propose a use of multiple turbo codes [22] to improve the performance between the source and relay. Direct application of multiple turbo codes, however, results in coding rate lower than $1 / 3$ and thus reduces the transmission rate (or bandwidth efficiency). To overcome this, we further propose the use of conventional puncturing applied at the source transmission as shown in Fig. 4. This operation does not reduce the diversity order at the destination since the source always transmits all the information sequence and thus (9) still holds. Consequently, even if the coding rate of the mother code is set to be $R=1 / 4$, the resulting coding rate after conventional puncturing is $R_{c}=1 / 2$. 


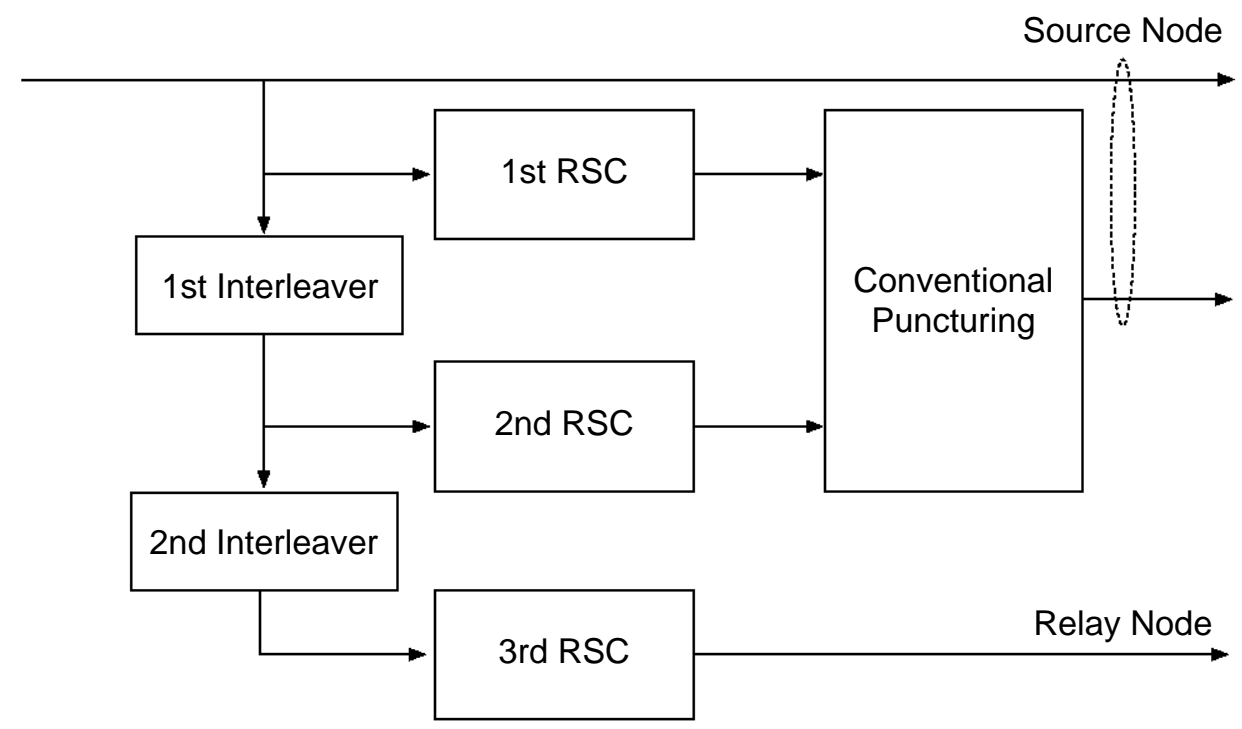

Fig. 4. Equivalent encoding model of multiple turbo code for the dynamic coded cooperation.

\section{Receiver Structure}

The receiver configuration and its decoding process for the proposed dynamic coded cooperation are described here. For powerful class of channel code such as turbo codes, the decoder typically employs the iterative a posterior probability (APP) algorithm. The APP decoder generates a hard decision and soft output in the form of APP on the basis of the received sequence. It computes the log-likelihood ratio

$$
\Lambda\left(u_{t} \mid \mathbf{Y}\right)=\log \left(\frac{\operatorname{Pr}\left(u_{t}=1 \mid \mathbf{Y}, h_{s d}, h_{r d}\right)}{\operatorname{Pr}\left(u_{t}=0 \mid \mathbf{Y}, h_{s d}, h_{r d}\right)}\right),
$$

for all $t$, where $t$ denotes the ordering of the information, $u_{t}$ is the information bit, and $\mathbf{Y}$ indicates the sequence of the detector outputs. Finally, a hard decision $\hat{u}_{t}$ is made by

$$
\hat{u}_{t}= \begin{cases}0, & \text { if } \Lambda\left(u_{t} \mid \mathbf{Y}\right) \leq 0 \\ 1, & \text { else. }\end{cases}
$$

Metric calculations and decoding at the relay are given similar to the operations of the destination. Thus, we only describe the procedures at the destination in the following. Decoding at the destination can be performed easily since this channel can be considered as a simple single-input single-output (SISO) or multiple-input single-output (MISO) channel. The receiver structure is depicted in Fig. 5. After receiving the signals, power detector detects the length 


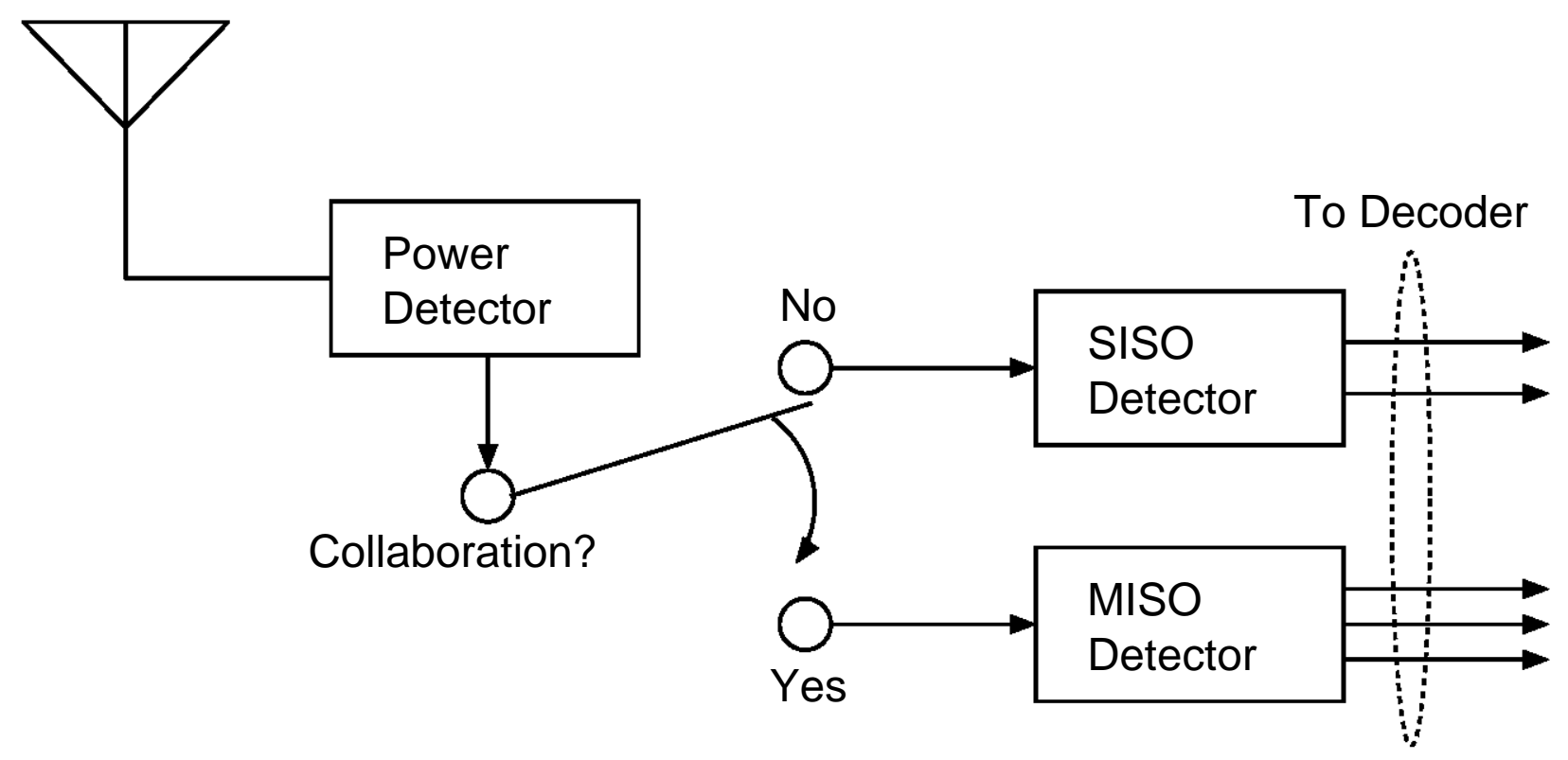

Fig. 5. Block diagram of a detector for the proposed method.

of $n_{1}^{\prime}$ [12]. Then, corresponding bit metrics are calculated by using SISO and MISO detectors based on the knowledge of $n_{1}^{\prime}$. During the listening phase, the metric for the $i$ th received signal denoted by (2) is given by

$$
\lambda\left(t, x_{s}(i)\right)=\exp \left(-\frac{\left|y_{s d}(i)-h_{s d} x_{s}(i)\right|^{2}}{2 \sigma^{2}}\right),
$$

where $|\cdot|$ denotes a magnitude of a complex number.

During the cooperation phase, the received signal can be written by (4) and thus the metric for the $i$ th received signal $y_{c}(i)$ and transmitted signal $x_{s}(i)$ is given by

$$
\lambda\left(t, x_{s}(i)\right)=E_{x_{r}(i)}\left[\exp \left(-\frac{\left|y_{c}(i)-h_{s d} x_{s}(i)-h_{r d} x_{r}(i)\right|^{2}}{2 \sigma^{2}}\right)\right],
$$

where $E[\cdot]$ indicates the expectation, and that for $x_{r}(i)$ is given by

$$
\lambda\left(t, x_{r}(i)\right)=E_{x_{s}(i)}\left[\exp \left(-\frac{\left|y_{c}(i)-h_{s d} x_{s}(i)-h_{r d} x_{r}(i)\right|^{2}}{2 \sigma^{2}}\right)\right] .
$$

After the metrics calculations, the decoder attempts to decode the information based on iterative decoding fashion. Overall decoder process at the destination is illustrated in Fig. 6, where $\pi_{i j}$ indicates the permutation of the time-ordering from $i$ th decoder to $j$ th decoder. Note that the probabilities in the operation of the expectation in the above equations may be calculated by 


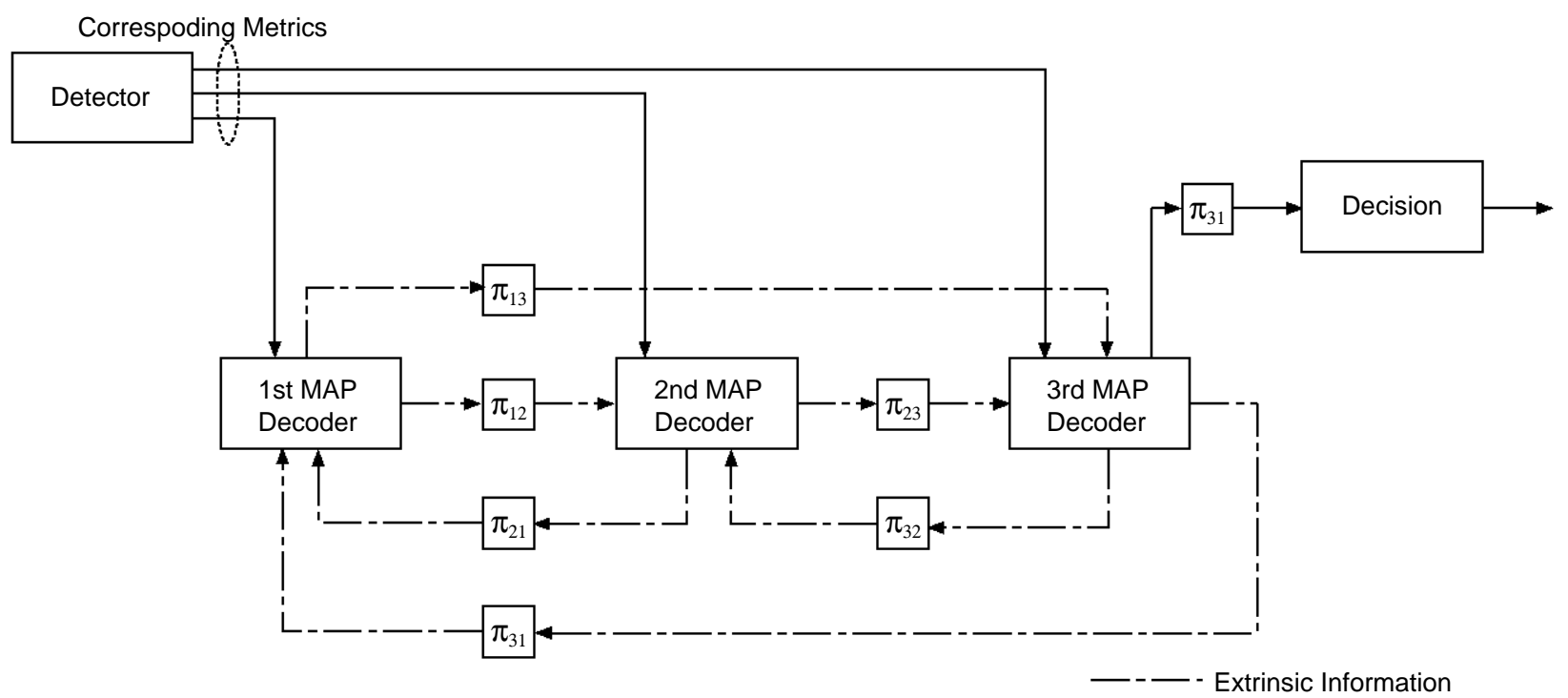

Fig. 6. Block diagram of a decoder for the proposed method where $\pi_{i j}$ indicates the permutation of time-ordering from $i$ th decoder to $j$ th decoder.

using extrinsic information. Also, the decision feedback approach such as BICM-ID may further improve the performance [23]. However, these outer iteration approaches increase the complexity of decoder noticeably. In the following, uniform probability is assumed in the calculation of expectation. Thus, extrinsic information generated by the decoders is not passed to the detector.

\section{Design of Power Detector For Dynamic Coded CoOperation}

In dynamic coded cooperation, the relay can dynamically choose its own coding rate, i.e., $n_{1}^{\prime}$, by being aware of its own receiving channel quality. Therefore, the destination also should cognize (i.e., estimate) the duration of listening phase chosen by the relay. In this section, the estimation method of $n_{1}^{\prime}$ is studied based on the simple power detector, which is often used in cognitive radio networks to detect existences of other devices or systems [3]. Note that we omit the effect of the process delay $\Delta n$, i.e., $\Delta n=0$, in this section for simplicity of notation.

\section{A. Derivation of Probability Density Function of Power Detector Output}

The power detector should detect whether the relay is in the cooperation phase or not for each $n_{b}$ received symbols (i.e., one fraction). The power detector outputs the summation of the power of $n_{b}$ received signals. Design of an optimal power detector requires a knowledge of its 
statistical property. Therefore, in what follows, we derive the probability density function (pdf) of power detector outputs.

1) Probability Density Function (PDF) of Power Detector Output in Listening Phase: The received signal in the listening phase is given by (2). Then, the $l$ th output of power detector from the $l$ th received fraction is written as

$$
Y(l)=\sum_{i=k+1+n_{b} l}^{k+n_{b}(l+1)}\left|y_{s d}(i)\right|^{2} .
$$

Also, the total power of $n_{b}$ transmitted signals $q_{1}(l)$ in the $l$ th fraction is given by

$$
\begin{aligned}
q_{1}(l) & =\sum_{i=k+1+n_{b} l}^{k+n_{b}(l+1)}\left|h_{s d} x_{s}(i)\right|^{2} \\
& =n_{b}\left|h_{s d}\right|^{2} .
\end{aligned}
$$

Then, the pdf of $Y$ is given by the noncentral chi-square pdf [24]. Dropping the time index $l$, the pdf can be given by

$$
p\left(Y \mid q_{1}\right)=\frac{1}{2 \sigma^{2}}\left(\frac{Y}{q_{1}}\right)^{\left(n_{b}-1\right) / 2} e^{-\left(q_{1}+Y\right) / 2 \sigma^{2}} I_{n_{b}-1}\left(\sqrt{Y q_{1}} \frac{1}{\sigma^{2}}\right),
$$

where $I_{m}(x)$ is the $m$-th order modified Bessel function of the first kind.

2) Probability Density Function (PDF) of Power Detector Output in Cooperation Phase: The received signal in the cooperation phase is given by (4). Then, the lth output of the power detector from the $l$ th received fraction is given by

$$
Y(l)=\sum_{i=k+1+n_{b} l}^{k+n_{b}(l+1)}\left|y_{c}(i)\right|^{2} .
$$

Let $q_{2}(l)$ denote the total power of $n_{b}$ superposed signals received from the source and the relay given by

$$
q_{2}(l)=\sum_{i=k+1+n_{b} l}^{k+n_{b}(l+1)}\left|h_{s d} x_{s}(i)+h_{r d} x_{r}(i)\right|^{2} .
$$

Without loss of generality, we hereafter drop the time index $l$. Then, if the knowledge of $q_{2}$ is available at the destination, the exact pdf of $Y$ in the cooperation phase can be simply derived by replacing $q_{1}$ with $q_{2}$ in (19). However, it is infeasible to obtain the knowledge of $q_{2}$ in practice since it depends on the two transmitted sequences. Thus, we utilize the following approximation:

$$
q_{2} \approx n_{b}\left(\left|h_{s d}\right|^{2}+\left|h_{r d}\right|^{2}\right)
$$


Consequently, the pdf in the cooperation phase is approximately written as

$$
p\left(Y \mid q_{2}\right)=\frac{1}{2 \sigma^{2}}\left(\frac{Y}{q_{2}}\right)^{\left(n_{b}-1\right) / 2} e^{-\left(q_{2}+Y\right) / 2 \sigma^{2}} I_{n_{b}-1}\left(\sqrt{Y q_{2}} \frac{1}{\sigma^{2}}\right) .
$$

\section{B. Derivation of Decision Metric for Power Detector}

Based on the pdfs derived in the previous subsection, the power detector can decide whether the relay is in the cooperation phase or not. We here derive the simple and practical decision metric for the power detector. The log-likelihood ratio of two situations is given by

$$
\Lambda(Y) \triangleq \log \left[\frac{p\left(Y \mid q_{1}\right)}{p\left(Y \mid q_{2}\right)}\right] \underset{q_{2}}{\stackrel{q_{1}}{\gtrless}} 0 .
$$

From (19) and (23), the log-likelihood ratio can be rewritten as

$$
\log \left[\frac{I_{n_{b}-1}\left(\frac{\sqrt{Y q_{1}}}{\sigma^{2}}\right)}{I_{n_{b}-1}\left(\frac{\sqrt{Y q_{2}}}{\sigma^{2}}\right)}\right] \underset{q_{2}}{\stackrel{q_{1}}{\gtrless}} \frac{q_{1}-q_{2}}{2 \sigma^{2}}-\left(n_{b}-1\right) \log \sqrt{\frac{q_{2}}{q_{1}}} .
$$

Unfortunately, direct calculation of (25) may be complex, since the calculation of the Bessel function is necessary. Therefore, we utilize an exponent-form approximation of the Bessel function as

$$
I_{m}(x) \approx \frac{1}{\sqrt{2 \pi x}} \exp (x)
$$

The above approximation may become accurate for $x \gg m$ [25]. Note that this condition holds unless $q_{2}$ is infinitesimally small. However, once the magnitude of both channel coefficients in $q_{2}$ is approximately zero, the decoder cannot decode the information even if the power detector correctly detects the relay's transmission. Thus, we restrict our attention to the case where the above condition holds. Then, substituting above approximation with the Bessel functions in (25) and with some manipulations that do not affect the decision, the log-likelihood ratio can be expressed as

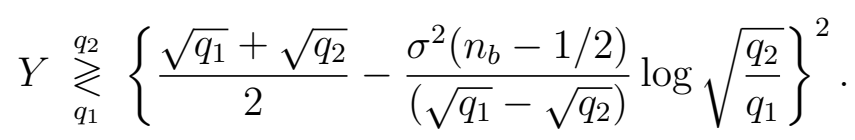

By using (27), the destination can be aware of the duration of the listening phase. For simplicity of the receiver circuit at the destination, we assume that once the power detector decides that the current fraction is in the cooperation phase, the power detector does not perform the detection of $n_{1}^{\prime}$ for subsequent fractions. 


\section{Performance Analysis of The Proposed Power Detector}

Based on the above decision metric, we analyze the asymptotic error probability of the proposed power detector to show that its performance depends on the length of fraction $n_{b}$ and thus the fractional reception mitigates the difficulty of the cognition of $n_{1}^{\prime}$ at the destination. To calculate the error probability, the cumulative density function (cdf) of the noncentral chisquare pdf is required, which can be expressed in terms of the generalized Marcum-Q function [24]:

$$
Q_{m}(a, b)=\int_{b}^{\infty} x\left(\frac{x}{a}\right)^{m-1} e^{-\left(x^{2}+a^{2}\right) / 2} I_{m-1}(a x) d x
$$

where $m>0$ and $0<a, b<\infty$.

Then, the error probability of the power detector is given by

$$
P_{b}=\frac{1}{2}\left\{1+Q_{n_{b}}\left(\frac{\sqrt{q_{1}}}{\sigma}, \beta\right)-Q_{n_{b}}\left(\frac{\sqrt{q_{2}}}{\sigma}, \beta\right)\right\},
$$

where

$$
\beta \triangleq \frac{\sqrt{q_{1}}+\sqrt{q_{2}}}{2 \sigma}-\frac{\sigma\left(n_{b}-1 / 2\right)}{\left(\sqrt{q_{1}}-\sqrt{q_{2}}\right)} \log \sqrt{\frac{q_{2}}{q_{1}}} .
$$

Note that the above equation does not express the exact error probability of the power detector since the pdf corresponding to the cooperation phase is not exact. Also, unfortunately, it is difficult to perform further simplification of (29) and thus we consider its asymptotic behavior in high SNR region. Generalized Marcum-Q function has the following properties:

$$
Q_{m}(a, \infty)=0
$$

where $0<a<\infty$ and

$$
Q_{m}(\infty, b)=1
$$

where $0<b<\infty$. Thus, the difference between the first and second arguments of Marcum-Q function defines the value of convergence. In sufficiently high SNR region, the second term of $\beta$ in (30) may converge with zero. Thus, we utilize the following approximation as

$$
\beta \approx \frac{\sqrt{q_{1}}+\sqrt{q_{2}}}{2 \sigma}
$$


Then, the difference between the first and second arguments of the former Marcum-Q function in (29) can be given by

$$
\begin{aligned}
\frac{\sqrt{q_{1}}}{\sigma}-\beta & \approx \frac{1}{2 \sigma}\left(\sqrt{q_{1}}-\sqrt{q_{2}}\right) \\
& =\frac{n_{b}}{2 \sigma}\left\{\left|h_{s d}\right|-\sqrt{\left|h_{s d}\right|^{2}+\left|h_{r d}\right|^{2}}\right\}<0 .
\end{aligned}
$$

On the other hand, the difference between the first and second arguments of the latter Marcum-Q function in (29) can be also given by

$$
\begin{aligned}
\frac{\sqrt{q_{2}}}{\sigma}-\beta & \approx \frac{1}{2 \sigma}\left(\sqrt{q_{2}}-\sqrt{q_{1}}\right) \\
& =\frac{n_{b}}{2 \sigma}\left\{\sqrt{\left|h_{s d}\right|^{2}+\left|h_{r d}\right|^{2}}-\left|h_{s d}\right|\right\}>0 .
\end{aligned}
$$

Hence, the former Marcum-Q function in (29) will converge with zero and the latter will converge with one. Consequently, the error probability of the power detector should converge with zero for high SNR. Moreover, these differences are proportional to the length of the fraction $n_{b}$. This means that the convergence rate of Marcum-Q functions may depend on $n_{b}$ and thus the error probability of the power detector also depends on the length of the fraction $n_{b}$, i.e., the number of fractions $K$. Therefore, increasing the length of the fraction $n_{b}$ (i.e., decreasing the number

of the fractions $K$ ) decreases the probability of inaccurate cognition of $n_{1}^{\prime}$ at the destination whereas the number of selectable coding rate at the relay is also decreased.

\section{NumericAl Results}

In this section, we show some numerical results to justify the code design for the proposed cooperation and demonstrate the performance of the proposed approach with the simple power detector in practical scenarios.

\section{A. Effect of Code Design on Achievable Diversity Order}

We start with the comparison between the conventional and component puncturing in order to justify the analysis in Section III-B. The coding rate of the mother code is $1 / 3$, the component code is the rate $1 / 2 \mathrm{RSC}$ code $(37,21)_{8}$ in octal form, and the random interleaver is assumed. The number of iteration is 10 and the information size is 3992 bits. Also, quadrature phase shift keying (QPSK) is used for simulations, i.e., $M=4$, in the rest of the paper. The coding rate 


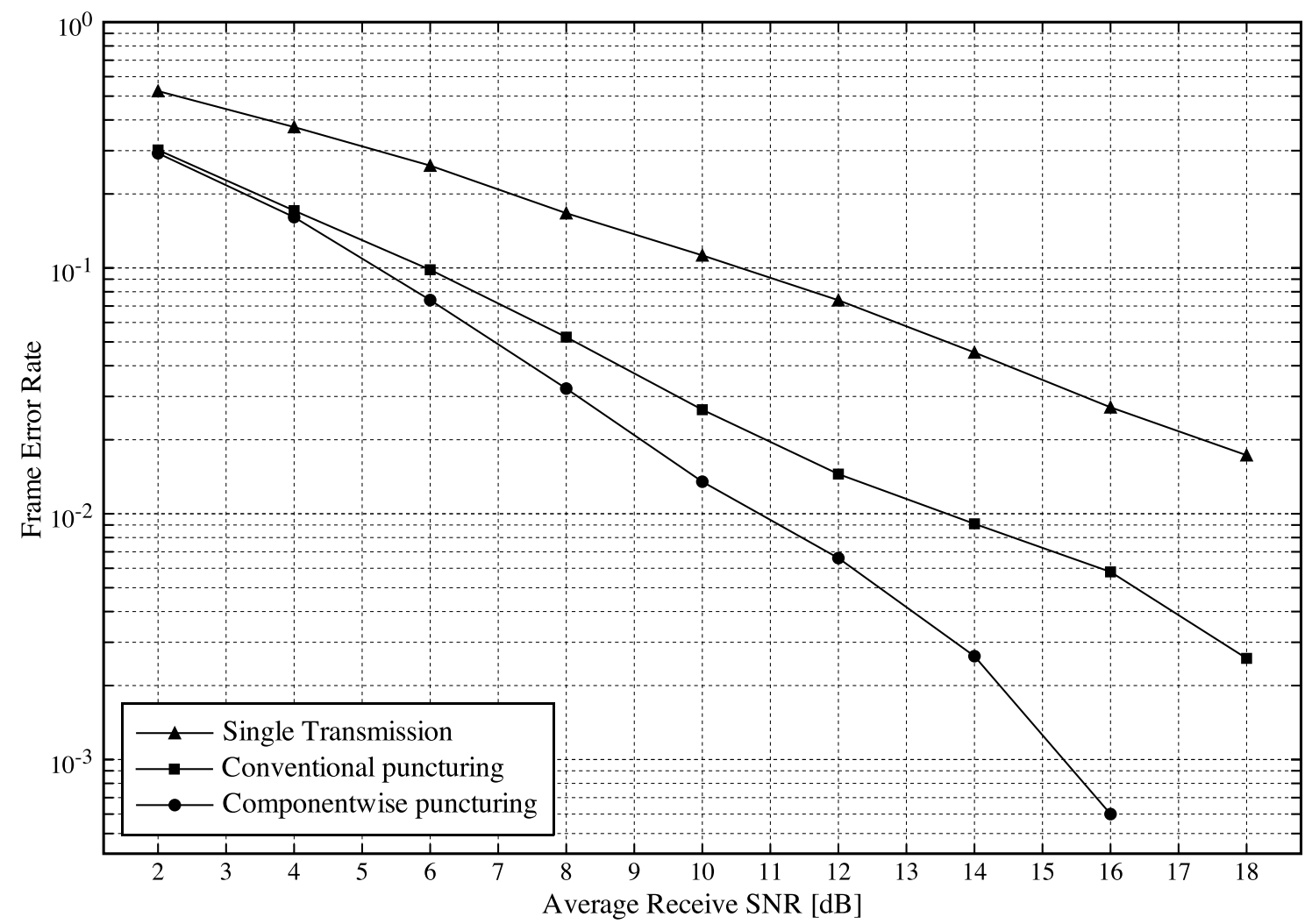

Fig. 7. FER performances of conventional puncturing and componentwise puncturing with orthogonal channels.

of the source is chosen as half and thus the length of the frame is 4000 symbols (8000 bits) because of 8 trellis termination bits. Therefore, additional redundant bits transmitted by the relay is 2000 symbols (4000 bits). We emphasize that the purpose of this subsection is to confirm the validity of full diversity condition developed in Section III-B. Therefore, only in this subsection, we assume that the source and relay use separate (i.e., orthogonal) channels. Also for the above reason, the error-free transmission is assumed between the source and the relay and the effect of process delay is not considered here (i.e., $\Delta n=0$ ).

Figure 7 shows the frame error rate (FER) performance of componentwise puncturing, conventional puncturing, and single transmission with the rate half turbo code. As observed, the conventional puncturing cannot achieve full diversity order of two even though the performance is improved compared with the single transmission. This is due to the fact that the conventional puncturing has some error events with incomplete diversity as shown in Section III-B. As a 


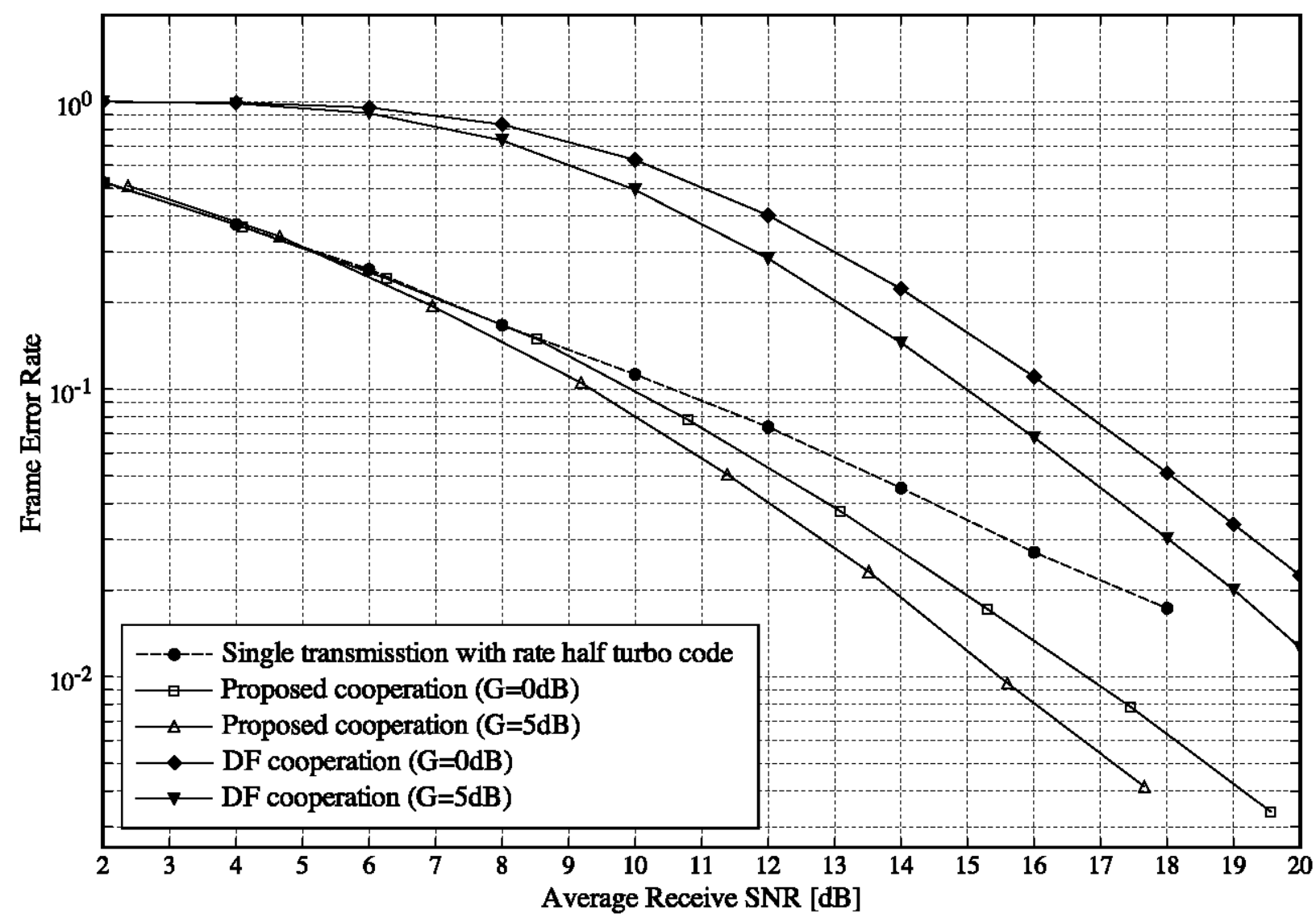

Fig. 8. FER performances of single transmission, conventional decode-and-forward (DF) cooperation, and proposed dynamic coded cooperation, where $G=0 \mathrm{~dB}$ and $5 \mathrm{~dB}$.

result, the performance of the componentwise puncturing is superior to that of conventional puncturing since the former puncturing always achieves full diversity.

\section{B. Performance of the Dynamic Coded Cooperation}

We here show the performance of the dynamic coded cooperation with perfect knowledge of $n_{1}^{\prime}$ by comparing with conventional DF cooperation. Following $[12,16]$, we here define the average receive SNR at the destination for the proposed approach as

$$
\tilde{\Gamma}=\left(2-\frac{\overline{n_{1}^{\prime}}}{n}\right) \Gamma_{s d}
$$

where $\overline{n_{1}^{\prime}}$ denotes the average cooperation level averaged over the ensemble of channel realizations. Most of the parameters are identical with those in the previous subsection whereas the coding rate of the mother code is assumed to be $1 / 4$ and the information size is 3988 bits. 
Also, the coding rate of the source is chosen as half and thus the length of the frame is 4000 symbols ( 8000 bits) for the proposed approach because of 12 trellis termination bits. The number of fractions $K$ is given as 4 for dynamic coded cooperation. From this subsection we no longer assume an ideal separate channel model; and the same channel is shared by the source and relay as in [12]. However, the effect of process delay is still not considered here (i.e., $\Delta n=0$ ). In order to evaluate the performance of the proposed approach, turbo coded single transmission and uncoded DF cooperation are considered as benchmarks. For a single transmission, the source transmits the rate half turbo coded information with conventional puncturing. Note that these approaches have identical bandwidth efficiency compared with the proposed approach.

Figure 8 shows the FER performance of single transmission, DF cooperation, and the proposed coded cooperation over the block Rayleigh fading channel. To investigate the effect of the proximity, relative path-loss gains, $G=0 \mathrm{~dB}$ and $5 \mathrm{~dB}$, are considered. It is observed that both proposed coded cooperation and DF cooperation achieve the diversity order of two, i.e., full diversity, in high SNR region. Furthermore, the proposed coded cooperation improves about $6 \mathrm{~dB}$ at a FER of $5.0 \times 10^{-2}$ compared with the DF cooperation regardless of path-loss gain $G$. Moreover, it is worth mentioning that the proposed cooperation with $G=5 \mathrm{~dB}$ rapidly achieves the maximum gradient because of dynamic adaptation of adequate coding rate. Thus, the proposed coded cooperation can exploit the proximity of the relay without loss of bandwidth efficiency.

\section{Performance Comparison with Conventional Coded Cooperation}

We compare the performance of the dynamic coded cooperation with that of the conventional coded cooperation $[9,11]$. Note that we do not use the conventional puncturing at the source for the dynamic coded cooperation in order to achieve the identical bandwidth efficiency compared with the conventional coded cooperation and thus $R_{c}=1 / 3$. Also, the other parameters are identical with those in Section V-B.

Figure 9 shows the FER performances of single transmission, conventional coded cooperation, and the proposed dynamic coded cooperation in the case of $G=0 \mathrm{~dB}$ and $5 \mathrm{~dB}$ where $n_{1}^{\prime}$ is assumed to be known at the destination. As observed, the performance gain of the proposed 


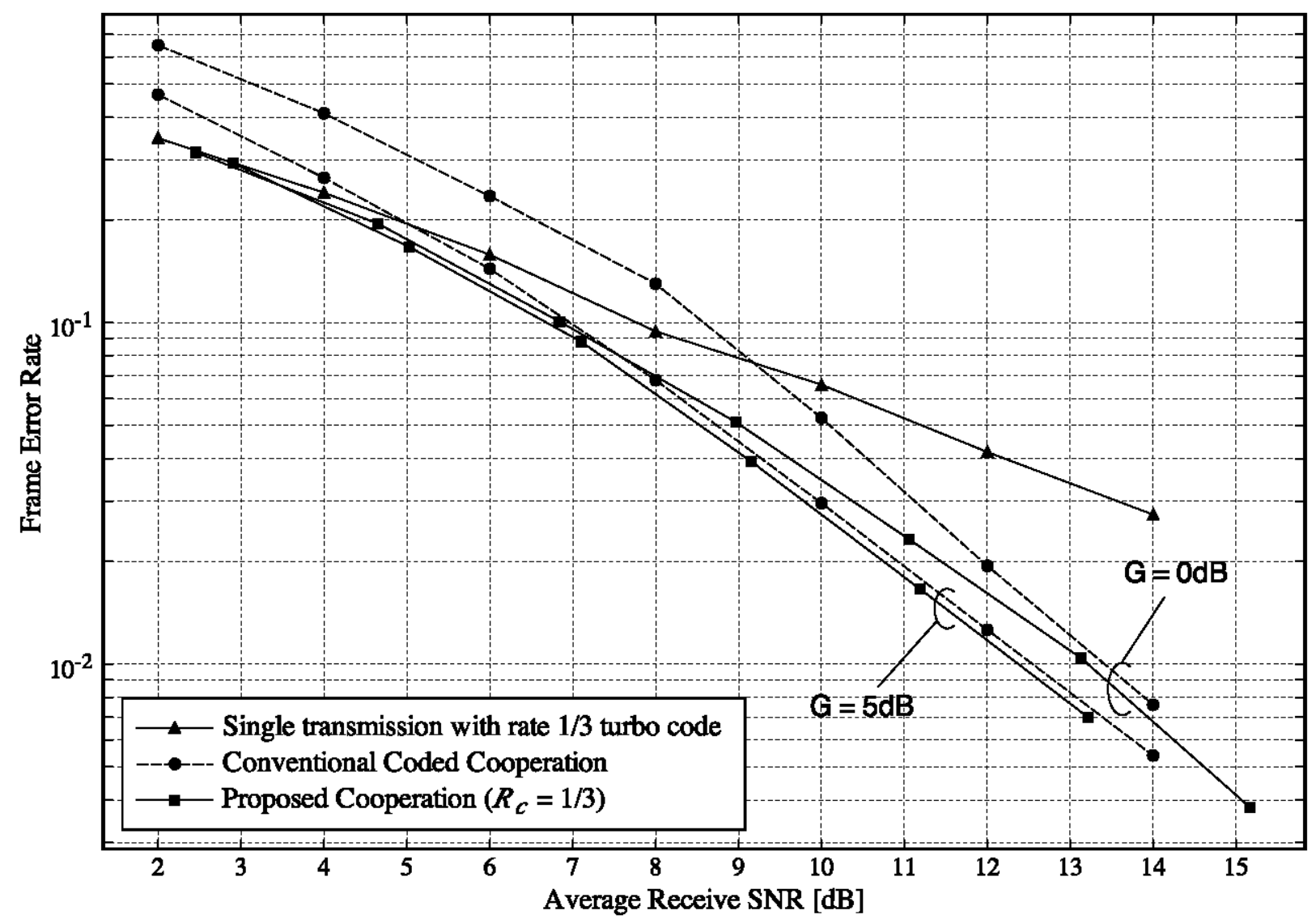

Fig. 9. FER comparison of conventional turbo coded cooperation and proposed dynamic coded cooperation without conventional puncturing at the source (i.e., $R_{c}=1 / 3$ ), where $G=0 \mathrm{~dB}$ and $5 \mathrm{~dB}$.

cooperation is noticeable when all channels in the network have low average SNR. Specifically, when $G=0 \mathrm{~dB}$, it is about $2 \mathrm{~dB}$ compared with the conventional coded cooperation at a FER of $1.0 \times 10^{-1}$. This significant performance improvement stems from the dynamic adaptation of the coding rate between the source and the relay and increased coding gain because of using multiple turbo code. This performance gap shows the advantage of using the proposed approach since it can be conjectured that the proposed approach can achieve the superior performance even with the low instantaneous SNR between the source and the relay.

\section{The Effect of Cognition of $n_{1}^{\prime}$}

We consider the performance of dynamic coded cooperation with the effect of cognition of $n_{1}^{\prime}$. Figure 10 shows the error probability of the power detector proposed in Section IV over 


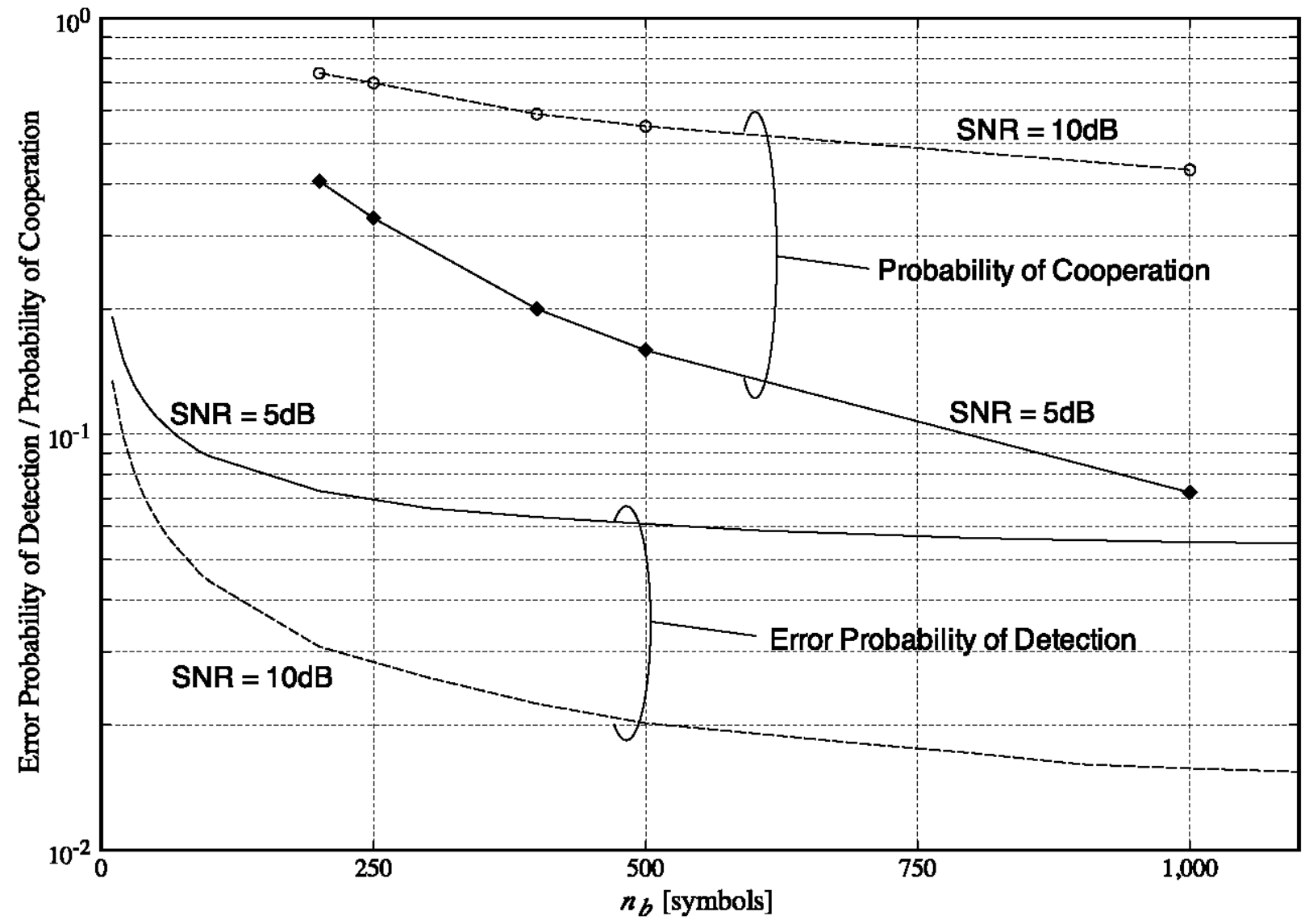

Fig. 10. Error probability performance of the proposed power detector subject to the length of fraction $n_{b}$ over Rayleigh fading channel at $\mathrm{SNR}=5 \mathrm{~dB}$ and $10 \mathrm{~dB}$.

Rayleigh fading channel at $\mathrm{SNR}=5 \mathrm{~dB}$ and $10 \mathrm{~dB}$. Also, the probability of cooperation is shown in the figure, where parameters are assumed to be identical with those in Section V-B and thus the maximum number of symbols transmitted by the relay is 2000 symbols. As observed, by increasing the length of fraction $n_{b}$ the performance of the power detector is improved. Moreover, when $n_{b}$ is larger than about 500 symbols, the performance almost converges in both cases. On the other hand, the probability of the cooperation decreases as increasing $n_{b}$. This observation indicates that if larger $K$ is chosen, the relay can choose the arbitrary coding rate adequate to the instantaneous channel quality between the source and relay and thus this dynamic adaptation may improve the probability of the cooperation. This however leads to the performance degradation of the power detector since $K$ defines the length of the fraction $n_{b}$.

In Fig. 11, the FER performances of the dynamic coded cooperation with the power detector 


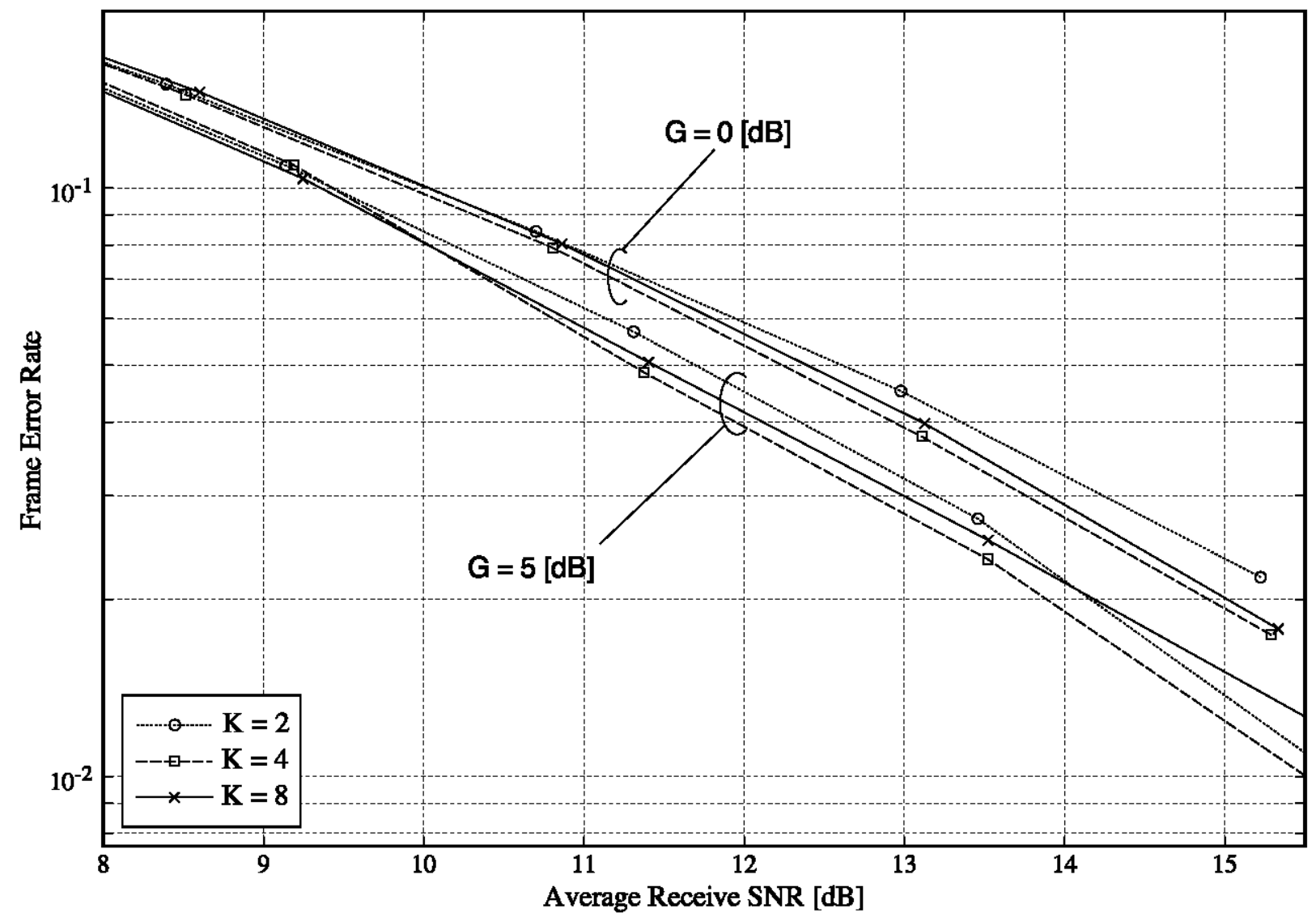

Fig. 11. FER performances of proposed coded cooperation with the power detection for various number of fractions $(K=2$, 4 , and 8).

are shown, where the parameters except for the number of fractions $K$ are assumed to be identical with those in Section V-B and the number of fractions is assumed to be $K=2$, 4 , and 8. Also, to investigate the effect of the proximity, relative path-loss gains, $G=0 \mathrm{~dB}$ and $5 \mathrm{~dB}$, are considered. From the figure, the performance with $K=4$ is always superior to that with the others. This observation may confirm the statements from Fig. 10 since the performance of the power detector almost converges when $n_{b}=500$ symbols (i.e., $K=4$ ) and also its corresponding probability of the cooperation is higher than that of $K=2$ (i.e., $n_{b}=1000$ ). Moreover, the performance of $K=4$ is almost identical with that with the perfect knowledge of $n_{1}^{\prime}$. Specifically, the required average receive SNR is about $15.5 \mathrm{~dB}$ at a FER of $1.0 \times 10^{-2}$ for both cases with $G=5 \mathrm{~dB}$ (see Fig. 8).

Interestingly, when $G=5 \mathrm{~dB}$, the performance with $K=2$ becomes superior to that with 
$K=8$ at about $\mathrm{SNR}=15.5 \mathrm{~dB}$ whereas it cannot overcome the performance with $K=8$ when $G=0 \mathrm{~dB}$. This is caused by the performance improvement of the power detector in the region where the relay almost always cooperates. In the case of $K=8$, the power detector cannot cognize the value of $n_{1}^{\prime}$ precisely and this erroneous detection leads to the decoding error at the destination. On the other hand, in low SNR region, the performance of $K=8$ is superior to that of $K=2$ since the overall performance is dominated by the improvement in term of cooperation probability, rather than the degradation in terms of power detection capability.

Therefore, the number of fractions should be carefully chosen upon designing the dynamic coded cooperation with the power detector. If $K$ is adequately chosen, dynamic coded cooperation with a simple power detector shows the beneficial performance.

\section{E. The Effect of Process Delay at Relay}

Finally, we show the effect of process delay $\Delta n$ to the overall performance of the dynamic coded cooperation. Without loss of generality, we here define process delay ratio (PDR) to the length of the fraction as

$$
\operatorname{PDR}=\frac{\Delta n}{n_{b}} \times 100[\%]
$$

Figure 12 shows the FER performances of the proposed approach with $\mathrm{PDR}=10,20,30$, and $0 \%$ (i.e., ideal case), where $G=0 \mathrm{~dB}$ and $5 \mathrm{~dB}$. The parameters except for the process delay are identical with those in Section V-B. Note that the ideal knowledge of $n_{1}^{\prime}$ is assumed to be available at the destination for simplicity. Also, the number of fractions $K$ is assumed to be 4. This comparison exemplifies that the FER performances with PDRs are noticeably degraded compared with the ideal case. Especially, the proposed approach even with a small PDR may reduce the diversity gain as observed in the figure since the proposed approach cannot transmit the entire codeword generated by the 3rd component encoder as mentioned in Section III-B. The performance labeled with null padding indicates the performance of the proposed approach with the simple null padding for $G=0 \mathrm{~dB}$ and $5 \mathrm{~dB}$, where PDR $=20 \%$. Obviously, the performance with the null padding can achieve the close-to-ideal performance. In this example, the loss of the bandwidth efficiency by using the null padding is only $2.5 \%$ compared with the ideal case whereas this loss becomes $33 \%$ if the conventional coded cooperation is used. Thus, 


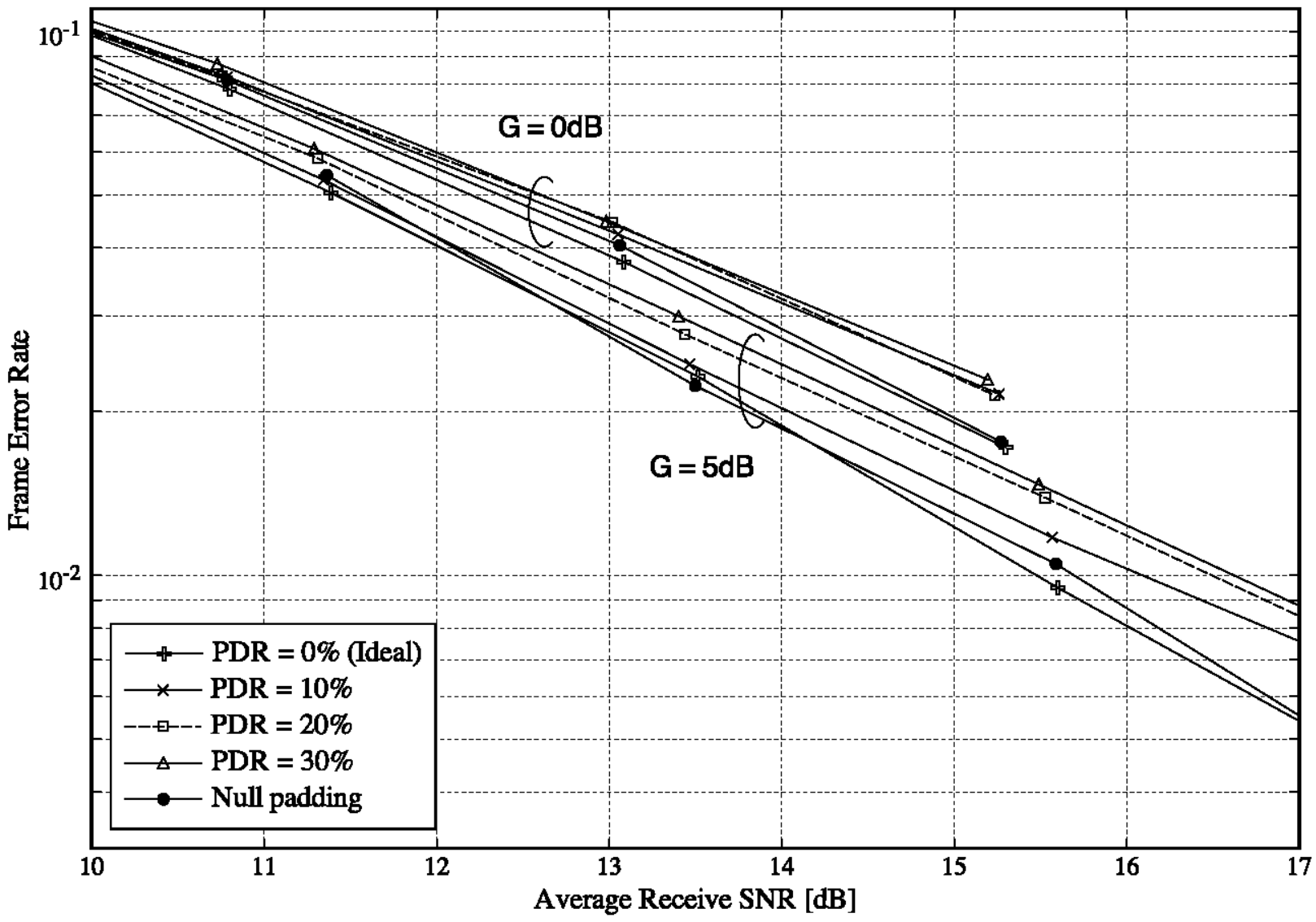

Fig. 12. FER performances of dynamic coded cooperation with the effect of process delay $\Delta n$, where $G=0 \mathrm{~dB}$ and $5 \mathrm{~dB}$.

even considering the effect of the PDR, the noticeable advantage of the proposed cooperation can be observed.

\section{Conclusions}

In this paper, we have proposed the practical dynamic coded cooperation based on the information theoretic framework proposed in [12] to exploit the limited transmission opportunities in cognitive radio networks. We have derived the design criterion of the rate compatible turbo code for the proposed approach to obtain the full diversity gain and proposed the use of multiple turbo code to improve probability of the cooperation. Also, the design of the power detector for the dynamic coded cooperation has been studied to autonomously recognize the relay's cooperation. The numerical results have shown that the proposed power detector can achieve the acceptable performance without increase of receiver complexity. Moreover, we have revealed that 
the process delay seriously affected the diversity gain at the destination and proposed the null padding as its simple countermeasure. Simulation results show the advantage of our proposed approach in terms of FER and bandwidth efficiency even considering the process delay.

\section{ACKNOWLEDGMENTS}

The authors wish to thank the Editor and anonymous reviewers for their comments that improved the quality of manuscript.

\section{REFERENCES}

[1] G. Staple and K. Werbach, "The end of spectrum scarcity," IEEE Spectrum, vol. 41, pp. 48-52, Mar. 2004.

[2] J. Mitola and G. Maguire, "Cognitive radio: Making software radios more personal," IEEE Pers. Commun., vol. 6, pp. 1318, Aug. 1999.

[3] S. Haykin, "Cognitive radio: Brain-empowered wireless communications," IEEE J. Select. Areas Commun., vol. 23, pp. 201220, Feb. 2005.

[4] Q. Zhang, J. Jia, and J. Zhang, "Cooperative relay to improve diversity in cognitive radio networks," IEEE Commun. Mag., vol. 47, pp. 111-117, Feb. 2009.

[5] S. M. Alamouti, "A simple transmit diversity for wireless communications," IEEE J. Select. Areas Commun., vol. 16, pp. 1451-1458, Oct. 1998.

[6] V. Tarokh, N. Seshadri, and A. R. Calderbank, "Space-time codes for high data rate wireless communication: Performance criterion and code construction," IEEE Trans. Inform. Theory, vol. 44, pp. 744-765, Mar. 1998.

[7] J. N. Laneman and G. W. Wornell, "Distributed space-time-coded protocols for exploiting cooperative diversity in wireless networks," IEEE Trans. Inform. Theory, vol. 49, pp. 2415-2425, Oct. 2003.

[8] J. N. Laneman, D. N. C. Tse, and G. W. Wornell, "Cooperative diversity in wireless networks: Efficient protocols and outage behavior," IEEE Trans. Inform. Theory, vol. 50, pp. 3062-3080, Dec. 2004.

[9] B. Zhao and M. C. Valenti, "Distributed turbo-coded diversity for the relay channel," Electron. Lett., vol. 39, pp. 786-787, May 2003.

[10] R. Liu, P. Spasojević, and E. Soljanin, "Incremental redundancy cooperative coding for wireless networks: Cooperative diversity, coding, and transmission energy gains," IEEE Trans. Inform. Theory, vol. 54, pp. 1207-1224, Mar. 2008.

[11] M. Janai, A. Hedayat, T. E. Hunter, and A. Nosratinia, "Coded cooperation in wireless communications:Space-time transmission and iterative decoding," IEEE Trans. Signal Processing, vol. 52, pp. 362-371, Feb. 2004

[12] P. Mitran, H. Ochiai, and V. Tarokh, "Space-time diversity enhancements using collaborative communications," IEEE Trans. Inform. Theory, vol. 51, pp. 2041-2057, June 2005.

[13] K. Azarian, H. E. Gamal, and P. Schniter, "On the achievable diversity-multiplexing tradeoff in half-duplex cooperative channels," IEEE Trans. Inform., vol. 51, pp. 4152-4172, Dec. 2005.

[14] M. N. Khormuji and E. G. Larsson, "Cooperative transmission based on decode-and-forward relaying with partial repetition coding," IEEE Trans. Wireless Commun., vol. 8, pp. 1716-1725, Apr. 2009.

[15] J. Castura and Y. Mao, "Rateless coding for wireless relay channels," IEEE Trans. Wireless Commun., vol. 6, pp. 1638-1642, May 2007.

[16] A. Shokrollahi, "Raptor codes," IEEE Trans. Inform., vol. 52, pp. 2551-2567, June 2006.

[17] X. Liu and T. Lim, "Fountain codes over fading relay channels," IEEE Trans. Wireless Commun., vol. 8, pp. 3278-3287, June 2009.

[18] K. Kumar and G. Caire, "Coding and decoding for the dynamic decode and foward relay protocol," IEEE Trans. Inform., vol. 55, pp. 3186-3205, July 2009.

[19] K. B. Letaief and W. Zhang, "Cooperative communications for cognitive radio networks," in Proc. of the IEEE, vol. 97, pp. 878-893, May 2009.

[20] B. Vucetic and J. Yuan, Turbo Codes: Principles and Applications. Kluwer Academic Publishers, 2000.

[21] S. Benedetto and G. Montorsi, "Unveiling turbo-codes: Some results on parallel concatenated coding shemes," IEEE Trans. Inform. Theory, vol. 42, pp. 409-428, Mar. 1996.

[22] D. Divsalar and F. Pollara, "Turbo codes for deep-space communications," in TDA Progress Report 42-120, (Jet Propulsion Laboratory, Pasadena, CA), pp. 66-77, May 1995.

[23] X. Li and J. A. Ritcey, "Trellis-coded modulation with bit interleaving and iterative decoding," IEEE J. Select. Areas Commun., vol. 17, pp. 715-724, Apr. 1999. 
[24] J. G. Proakis, Digital Communications. McGraw-Hill, fourth ed., 2001.

[25] W. H. Press, S. A. Teukolsky, W. T. Vetterling, and B. P. Flannery, Numerical Recipes in C : the art of scientific computing. New york: Cambridge Univ. Press, second ed., 1992.

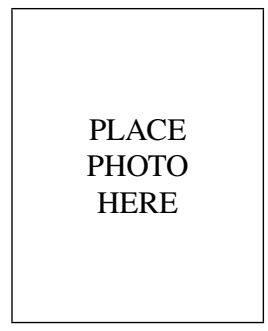

Koji Ishibashi (S'01-M'07) received the B.E. and M.E. degree in engineering from The University of Electro-Communications, Tokyo, Japan in 2002 and 2004 respectively. He received the Ph.D. degree in engineering from Yokohama National University, Yokohama, Japan in 2007.

Since April 2007, he has been with the Department of Electrical and Electronic Engineering, Shizuoka University, Hamamatsu, Japan, where he is currently an assistant professor.

His current research interests are wireless communications, cooperative communications, coded modulation, differential detection, and information theory.

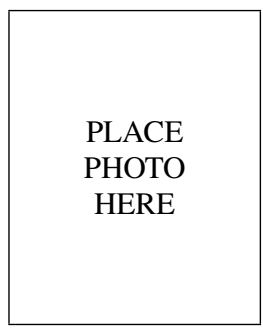

Koji Ishii (S'01-M'05) received the B.E., M.E., and Ph.D. degrees in electrical and computer engineering from Yokohama National University, Japan, in 2000, 2002, and 2005, respectively.

Since April 2005, he has been with the Department of Reliability-based Information Systems Engineering, Faculty of Engineering, Kagawa University, Japan, where he is currently a lecturer.

$\mathrm{He}$ is a member of IEEE communications and information theory society. Also, He is a member of IEICE and SITA, Japan.

His current research interests include wireless communications, information theory, iterative processing, multiple-input multiple-output (MIMO) systems, and cooperative communications.

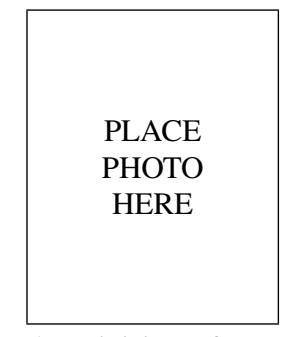

Hideki Ochiai (S'97-M'01) received the B.E. degree in communication engineering from Osaka University, Osaka, Japan, in 1996, and the M.E. and Ph.D. degrees in information and communication engineering from the University of Tokyo, Tokyo, Japan, in 1998 and 2001, respectively.

From 1994 to 1995, he was with the Department of Electrical Engineering, University of California, Los Angeles (UCLA), CA, under the scholarship of the Ministry of Education, Science, and Culture. From 2001 to 2003, he was a Research Associate at the Department of Information and Communication Engineering, the University of Electro-Communications, Tokyo, Japan. Since April 2003, he has been with the Department of Electrical and Computer Engineering, Yokohama National University, Yokohama, Japan, where he is currently an Associate Professor. From 2003 to 2004, he was a Visiting Scientist at the Division of Engineering and Applied Sciences, Harvard University, Cambridge, MA.

Dr. Ochiai currently serves as an Editor for IEEE TRANSACTIONS ON WIRELESS COMMUNICATIONS. 